\title{
Far Ultraviolet Spectroscopic Explorer (FUSE) observations of emitting and absorbing gas in the Local Interstellar Chimney
}

\author{
B. Y. Welsh ${ }^{1,2}$, S. Sallmen ${ }^{1}$, D. Sfeir ${ }^{3}$, R. L. Shelton ${ }^{4}$, and R. Lallement ${ }^{5}$ \\ ${ }^{1}$ Experimental Astrophysics Group, Space Sciences Laboratory, UC Berkeley, Berkeley, CA 94720, USA \\ 2 Eureka Scientific, 2452 Delmer Street, Oakland, CA 94602-3017, USA \\ ${ }^{3}$ Department of Aerospace and Mechanical Engineering, University of Southern California, Los Angeles, CA 90089, USA \\ ${ }^{4}$ Department of Physics and Astronomy, Johns Hopkins University, Baltimore, MD 21218, USA \\ 5 Service d'Aéronomie du CNRS, 91371 Verrières-le-Buisson, France
}

Received 12 March 2002 / Accepted 7 August 2002

\begin{abstract}
We present Far Ultraviolet Spectroscopic Explorer (FUSE) satellite measurements of the absorption and emission characteristics of interstellar gas associated with the Local Interstellar Chimney, which is an extension of the rarefied Local Bubble cavity that extends outward from the galactic disk towards the lower galactic halo. Far ultraviolet (FUV) diffuse background emission has been detected in the high ionization line of O VI $(\lambda 1032 \AA)$ for two lines-of-sight $\left(l=162.7^{\circ}, b=+57.0^{\circ}\right)$ and $\left(l=156.3^{\circ}, b=+57.8^{\circ}\right)$ at emission levels of $2500 \pm 700$ photons $\mathrm{cm}^{-2} \mathrm{~s}^{-1} \mathrm{sr}^{-1}$ (LU) and $3300 \pm 1100$ LU respectively. These levels of O VI emission are very similar to those found for four other lines-of-sight sampled thus far by the FUSE satellite, implying a fairly constant level of average O VI surface brightness emission at high galactic latitudes of about $2700 \mathrm{LU}$ with a standard deviation of $450 \mathrm{LU}$.

These emission-line data are supplemented by FUV interstellar absorption line measurements taken towards the hot DA white dwarf star, REJ $1032+532\left(l=157.5^{\circ}, b=+53.2^{\circ}\right)$, whose distance of $116 \mathrm{pc}$ places it within the Local Bubble region. No high ionization interstellar O VI $\lambda 1032 \AA$ absorption has been detected $\left(N(\mathrm{O}\right.$ VI $\left.)<13.0 \mathrm{~cm}^{-2}\right)$, which is consistent with the non-detections of interstellar C IV and Si IV absorption reported towards this star by Holberg et al. (1999a). Taken together, our FUV absorption and emission data may be explained by a scenario in which the O VI emission and absorption lines are both formed at the conductive interface of the neutral boundary to the Local Bubble. For the presently sampled sight-lines we have found no correlation between the OVI emission line intensity and the associated $0.25 \mathrm{keV}$ soft X-ray background flux as measured in the R1 and R2 bands by the ROSAT satellite. The OVI line intensities also show no correlation with the soft $\mathrm{X}$-ray background flux attributable to emission from the million degree $\mathrm{K}$ gas of the Local Hot Bubble as modeled by Kuntz \& Snowden (2000). Any (new) model of the Local Bubble must now be able to explain (i) the low levels of variability in both the O VI emission-line intensity and the associated soft X-ray background flux for galactic sight-lines $>|40|^{\circ}$, (ii) the observed pressure of $P / k \sim 10000 \mathrm{~cm}^{-3} \mathrm{~K}$ for the local hot interstellar gas, and (iii) the paucity of high ionization absorption lines observed within the local ISM and the sudden increase in their measured column density for distances beyond the Local Bubble neutral boundary.
\end{abstract}

Key words. ISM: atoms - ISM: bubbles - Galaxy: solar neighbourhood

\section{Introduction}

Although it has been over 40 years since the prediction (and subsequent discovery) of a hot $\left(T \sim 10^{6} \mathrm{~K}\right)$ component to the interstellar gas in our Galaxy (Spitzer 1956), we still know very little about the distribution, state or evolution of this hot plasma. Although several models for the origin of this gas have been proposed, which include (i) the cloud-evaporation model of McKee-Ostriker (1977), (ii) the galactic fountain model of Shapiro \& Field (1976), and (iii) the supernova bubble model of Cox \& Smith (1974), we note that no single model has yet

Send offprint requests to: $\mathrm{B}$. Y. Welsh, e-mail: bwelsh@ssl. berkeley .edu to be verified by current observations. This situation is exacerbated further by the fact that most of the major plasma cooling theories have also yet to be verified by observation, although we note that this may change in the near future with the anticipated launch of the NASA Cosmic Hot Interstellar Plasma Spectrometer (CHIPS) satellite in mid-2002 (Dixon et al. 1998) and the Korean-NASA SPEAR satellite in 2003 (Edelstein et al. 2000).

Our most detailed knowledge of hot interstellar (IS) gas in the galactic disk and halo has been deduced from far ultraviolet (FUV) absorption spectra of highly ionized species such as the doublet resonance lines of CIV $(\lambda 1550 \AA)$, SiIV $(\lambda 1394 \AA)$ and NV $(\lambda 1238 \AA)$. These ions sample interstellar gas with 
temperatures $\sim 60000 \mathrm{~K}-180000 \mathrm{~K}$ and all possess scaleheights in excess of $z>3 \mathrm{kpc}$ (Savage et al. 1997). It is probable that these line-species co-exist above the lower neutral halo (whose extent of $\sim 500 \mathrm{pc}$ is generally defined by a rarefied, warm neutral layer of gas called the "Lockman Layer", Lockman 1984) in a region of hot gas possibly advected from the galaxy by supernovae. However at present there is still no strong evidence to support any outflow of hot, ionized gas through a galactic fountain effect, and instead, halo observations of the C IV ion favor formation toward mostly negative velocities (Savage et al. 1997). Interestingly, the small value of $N(\mathrm{C}$ IV): $N(\mathrm{O}$ VI) found recently for low $z$ objects by Savage et al. (2000) is best explained by a conductive heating model in which the high ions are produced mainly in isolated low halo SNRs with hot gas properties similar to those of the ten million year old Local Bubble (LB) interstellar cavity. These conclusions have been supported by the recent observations of O VI ( $\lambda 1032,1038 \AA$ ) diffuse emission recorded in the high latitude sight-line towards $\left(l=113^{\circ}, b=+70.7^{\circ}\right)$ by Shelton (2002). These data suggest that most of the O VI emission (which samples gas with a temperature of $\sim 300000 \mathrm{~K}$ ) originates in the thick galactic disk or lower halo, and such gas may have been heated long ago rather than in a recent (SNR) shock event. We note, however, that the recent FUSE survey of O VI absorption in the galactic halo by Savage et al. (2001) reveals that gas parcels containing O VI are moving both towards and away from the plane with roughly equal frequency at velocities in excess of $|50| \mathrm{km} \mathrm{s}^{-1}$. These new FUSE data require a combination of models involving the radiative cooling of hot gas in a galactic fountain flow and the turbulent mixing of hot gases.

Although the distribution of both high and low ionization UV absorption lines towards many directions in the outer halo is well documented (Savage et al. 1997), conversely the region encompassing the high galactic disk and the beginning of the low inner halo gas $(z=0.2-0.5 \mathrm{kpc})$ is far less studied. Also, all previous selections of halo lines-of-sight have been made without prior knowledge of the intervening absorption contribution from both the ionized and neutral components of the LB cavity. Recently, Sfeir et al. (1999) have completed a survey of NaI (neutral gas) absorption for distances $<300 \mathrm{pc}$ of the Sun that has revealed an absorption boundary of cold and relatively dense, $N(\mathrm{HI})>5 \times 10^{19} \mathrm{~cm}^{-2}$, neutral gas surrounding the rarefied LB cavity with radii of between 65 to $250 \mathrm{pc}$. This interstellar cavity of low neutral gas density $\left(n_{\mathrm{H}}<0.01 \mathrm{~cm}^{-3}\right)$ has a well defined neutral boundary in the galactic plane, but at high galactic latitudes the LB appears to be partially openended in both hemispheres with no well-defined dense absorption boundary for $z<200 \mathrm{pc}$. This extension of the LB cavity as it begins to reach into the lower galactic halo has been confirmed by maps of the local distribution of EUV sources, such that this finger-like interstellar feature has been termed the "Local Chimney" (LC) (Welsh et al. 1999). In the northern galactic hemisphere the LC points in the general direction of Ursa Major towards $\left(l=155^{\circ}, b=+58^{\circ}\right)$ and has a diameter of $\sim 20^{\circ}$. This area of the sky contains the famous Lockman Hole line-of-sight, which is a small $4 \mathrm{deg}^{2}$ region centered on $\left(l=149^{\circ}, b=+54^{\circ}\right)$ with a low neutral HI column density of $4.5 \times 10^{19} \mathrm{~cm}^{-2}$ (Lockman et al. 1986). This general area is also associated with a significant enhancement of the $0.25 \mathrm{keV}$ soft X-ray background (SXRB) intensity as measured by the ROSAT satellite (Snowden et al. 1997). This region has been mapped in detail by Snowden et al. (1994), such that the enhanced SXRB flux in this direction can be attributed to an extragalactic component to the total measured soft X-ray flux. This low neutral-density IS cavity, perhaps extending as far as the lower halo region iteself, clearly provides a unique opportunity to sample local interstellar plasma over a long sight-line, as well as searching for any interaction between possible outflowing/inflowing LB gas and the local inner halo.

In this Paper we present both absorption and emission observations of interstellar plasma within the Local Chimney extension of the Local Bubble using the NASA Far Ultraviolet Spectroscopic Explorer (FUSE) satellite (Moos et al. 2000). Far UV absorption observations of the interstellar gas towards the hot white dwarf star REJ $1032+532(d=116$ pc $)$ are presented, together with supplementary FUSE observations of emitting gas from two very nearby regions within $5^{\circ}$ on the sky. By combining the presently derived characteristics of the absorbing and emitting gas along this local sight-line with 4 other sets of FUSE O VI observations, we are able to comment on the relation between the emission from the O VI $\lambda 1032 \AA$ line and the associated SXRB flux. These observations indicate that the level of diffuse emission from the O VI $\lambda 1032 \AA$ line is constant over the high galactic regions thus far sampled.

\section{Line-of-sight observations, data processing and reduction}

Spectral data were recorded during observations of three lines-of-sight along the Local Chimney direction of the local interstellar medium using the FUSE spectrograph (Sahnow et al. 2000). These observations consisted of one FUV absorption spectrum recorded towards the hot white dwarf star REJ $1032+532\left(\mathrm{Sp}=\mathrm{DA}, M_{v}=14.3, l=157.5^{\circ}, b=+53.2^{\circ}\right)$, and observations of the FUV background emission from two nearby sight-lines.

The absorption data taken towards REJ $1032+532$ were recorded in four separate integrations ( $7450 \mathrm{~s}$ total), with the spectral photon data being recorded in the detector histogram mode. Each of these data sets (which consist of 8 different spectral channels covering the $2912-1180 \AA$ range) was individually processed using version 1.8 .7 of the FUSE science data reduction (CALFUSE) pipeline, which corrects for geometric image distortions, background subtraction, image thermal drifts, detector deadtime, wavelength and flux calibration (Sahnow et al. 2000). These spectral channel data sets were subsequently co-added and averaged using standard IDL data reduction routines. Inspection of these averaged spectra revealed that data contained in the $\mathrm{LiF} 1 \mathrm{~A}, \mathrm{LiF} 1 \mathrm{~B}$ and $\mathrm{SiC} 2 \mathrm{~B}$ detector segments were of superior $S / N$ ratio, and these have been used in the subsequent absorption line analysis (although use was made of the other spectral channels as a confidence check on all subsequent line detections). The absorption spectra (shown in Fig. 1) were fairly well-exposed for wavelengths $>1000 \AA$ with typical $S / N$ ratios of $\sim 12$, whereas the spectra $<1000 \AA$ were of poor $S / N$ ratio $(<5)$. The FUSE instrument 


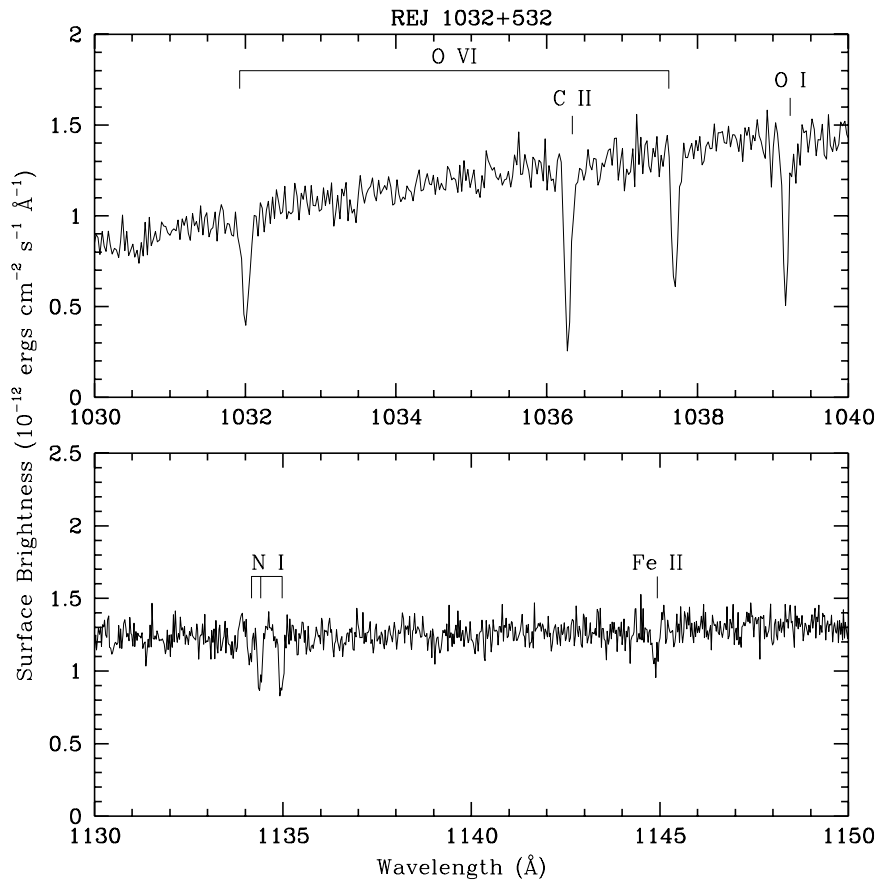

Fig. 1. Averaged FUSE absorption spectrum of the hot white dwarf REJ $1032+532$. The majority of interstellar lines detected towards this star are present in these two wavelength regions of the spectrum.

typically has an on-orbit velocity resolution of $\sim 13 \mathrm{~km} \mathrm{~s}^{-1}$, as determined from the fitting of weak interstellar absorption lines.

The two observations of the far UV background emission consisted of (i) a $38600 \mathrm{~s}$ integration, split into 16 exposures, that recorded diffuse emission from the galactic direction $(l=$ $\left.162.7^{\circ}, b=+57.0^{\circ}\right)$ - which we term sight-line "A" - which is a position some 20 arcsec away from the hot white dwarf star REJ $1043+490$ as listed by Vennes et al. (1997), and (ii) a $34100 \mathrm{~s}$ integration, split into 14 exposures, recording emission from $\left(l=156.3^{\circ}, b=+57.8^{\circ}\right)$, a position on the sky $\sim 18$ arcsec away from the hot white dwarf star REJ 1059+514 (which we term sight-line "B"). All data were taken with the FUSE LWRS aperture of size $30 \operatorname{arcsec} \times 30$ arcsec.

Both sets of diffuse background emission data, which were recorded in the time-tag detection mode, were processed using the full data sets, and also screened into night-only data. This screening, as well as event-burst removal, were performed using version 2.0.5 of the CALFUSE data processing pipeline. These data were further reduced to an extracted spectrum as described by Dixon et al. (2001), with the data being summed into 8 detector pixel bins $\left(\sim 15 \mathrm{~km} \mathrm{~s}^{-1}\right)$. For sight-line "A" we recorded $28.6 \mathrm{ksec}$ of night-only data and $10.0 \mathrm{ksec}$ of day-time data. For sight-line "B", $24.9 \mathrm{ksec}$ of night-only and $9.2 \mathrm{ksec}$ of day-time data were recorded. For sight-line "A" it was found that the best resultant $S / N$ spectra came from using the co-added (and exposure-weighted) day-time + night-only data (see Fig. 3). However, for sight-line "B" we present the night-only data, since addition of the day-time data resulted in a narrow emission (noise?) feature close to the O VI $\lambda 1032 \AA$ line that significantly reduced the resultant $S / N$ of the spectrum. A small number of off-axis photons associated with the
FUV continuum flux from the angularly close white dwarf star, REJ $1059+514$, also appeared on the detector, but these events were easily identifiable and did not contaminate the photon flux associated with the diffuse emission from sightline "B". Wavelengths for the emission line spectra (over the range $\lambda 1028-1040 \AA$ ) were derived from interpolating between nearby telluric emission lines of accurately known wavelengths. The spectral resolution of the FUSE instrument for observations of diffuse sources when used with the LWRS aperture is $\sim 100 \mathrm{~km} \mathrm{~s}^{-1}$, and the resulting absolute wavelength accuracy is typically $\pm 20 \mathrm{~km} \mathrm{~s}^{-1}$. All velocities in this paper are reported in the heliospheric frame of reference (for LSR velocities add $4 \mathrm{~km} \mathrm{~s}^{-1}$ ).

\section{Interstellar absorption line analysis}

The entire FUV spectrum of REJ $1032+532$ (1912-1180 ̊) was searched for the presence of interstellar absorption lines. We were guided in this search in two ways: (i) by reference to the list of FUV interstellar lines detected towards 4 white dwarfs in the local ISM by Jenkins et al. (2000), and (ii) by measurement of the relative wavelength shift between the observed FUSE line wavelength and the vacuum wavelengths of atomic lines. It was found that absorption feature detections fell into two groups, (a) those with wavelength shifts of $\sim+0.42 \AA$, and (b) those with shifts of $\sim+0.26 \AA$. The former set of lines were recognized as being stellar in origin (see the discussion of the O VI line later in this section), and the latter set were identified with an interstellar origin. Detections were only deemed valid if a line was present in more than one of the FUSE spectral channels, and these interstellar lines are listed in Table 1. In Fig. 1 we show two wavelength regions of the FUV absorption spectrum of REJ $1032+532$ that show the majority of the interstellar lines detected in this line-of-sight. We then fitted the local stellar continua around each interstellar absorption line with a multi-order polynomial to produce a residual line intensity profile, from which a total equivalent width $\left(W_{\lambda}\right)$ was determined. Each of these interstellar lines was then fit with one (or more) absorption components using the line-fitting procedure discussed in Sfeir et al. (1999). Each of these theoretical absorption profiles is characterized by 3 parameters: (i) a Gaussian velocity dispersion, $b$ value, (ii) an interstellar cloud component velocity, $V$, and (iii) a cloud component ion column density, $N$. Since the absolute wavelength solution to the FUSE data is not accurately known, we have assumed that the main absorption component of all the FUV interstellar lines occurs at the same velocity (i.e. $V_{\text {helio }}=+0.4 \mathrm{~km} \mathrm{~s}^{-1}$ ) as determined for the $16 \mathrm{UV}$ interstellar lines observed towards REJ 1032+532 by Holberg et al. (1999a) using the HST-STIS instrument. It was found that absorption from a single interstellar cloud was sufficient to produce adequate fits to the observed far UV profiles. The best-fit values of $b, V$ and $N$ for all the far UV interstellar lines are listed (together with their respective error estimates and equivalent width values) in Table 1, and the associated best-fit absorption profiles are shown in Fig. 2.

In Fig. 1 we note the detection of both $O$ VI ( $\lambda 1032$, $1038 \AA$ ) lines in absorption. However, we reject these lines as being of interstellar origin for the following 2 reasons. 
Table 1. Interstellar absorption lines towards REJ 1032+532.

\begin{tabular}{cccccc}
\hline \hline Line & $\begin{array}{c}W_{\lambda} \\
(\mathrm{m} \AA)\end{array}$ & $\begin{array}{c}V \\
\left(\mathrm{~km} \mathrm{~s}^{-1}\right)\end{array}$ & $b$ & $\begin{array}{c}N \\
\left(10^{12} \mathrm{at}^{-2} \mathrm{~cm}^{-2}\right)\end{array}$ & $S / N$ \\
\hline C II $\lambda 1036.3 \ldots$ & $78 \pm 8$ & $+0.4 \pm 0.7$ & $14.3 \pm 1$ & $\begin{array}{c}102 \pm 10 \\
<0.85\end{array}$ & 10 \\
C III $\lambda 977.0 \ldots$ & $<20$ & & & N/A & N/A \\
N I $\lambda 1134.2 \ldots$ & blended & +0.4 & N/A & $70 \pm 9$ & 11 \\
N I $\lambda 1134.4 \ldots$ & $21 \pm 3$ & $+0.4 \pm 2.5$ & $16.5 \pm 3$ & $72 \pm 8$ & 12 \\
N I $\lambda 1135.0 \ldots$ & $31 \pm 4$ & $+0.4 \pm 1.3$ & $15 \pm 2$ & $80 \pm 20$ & 5 \\
N II $\lambda 1084.0 \ldots$ & $69 \pm 10$ & $+0.4 \pm 1.0$ & $15 \pm 3$ & $600 \pm 200$ & 5 \\
O I $\lambda 988.8 / 988.6 \ldots$ & $140 \pm 15$ & $+0.4 \pm 1.5$ & $30 \pm 5$ & $640 \pm 75$ & 13 \\
O I $\lambda 1039.2 \ldots$ & $51 \pm 4$ & $+0.4 \pm 0.6$ & $11.2 \pm 1$ & 840 \\
O VI $\lambda 1031.9 \ldots$ & $<13$ & & & $<10$ & \\
Si II $\lambda 989.9 \ldots$ & $58 \pm 10$ & $+0.4 \pm 3.8$ & $27 \pm 5$ & $42 \pm 10$ & 5 \\
Fe II $\lambda 1144.9 \ldots$ & $29 \pm 6$ & $+0.4 \pm 5.0$ & $27 \pm 8$ & $27 \pm 5$ & 8 \\
\hline
\end{tabular}

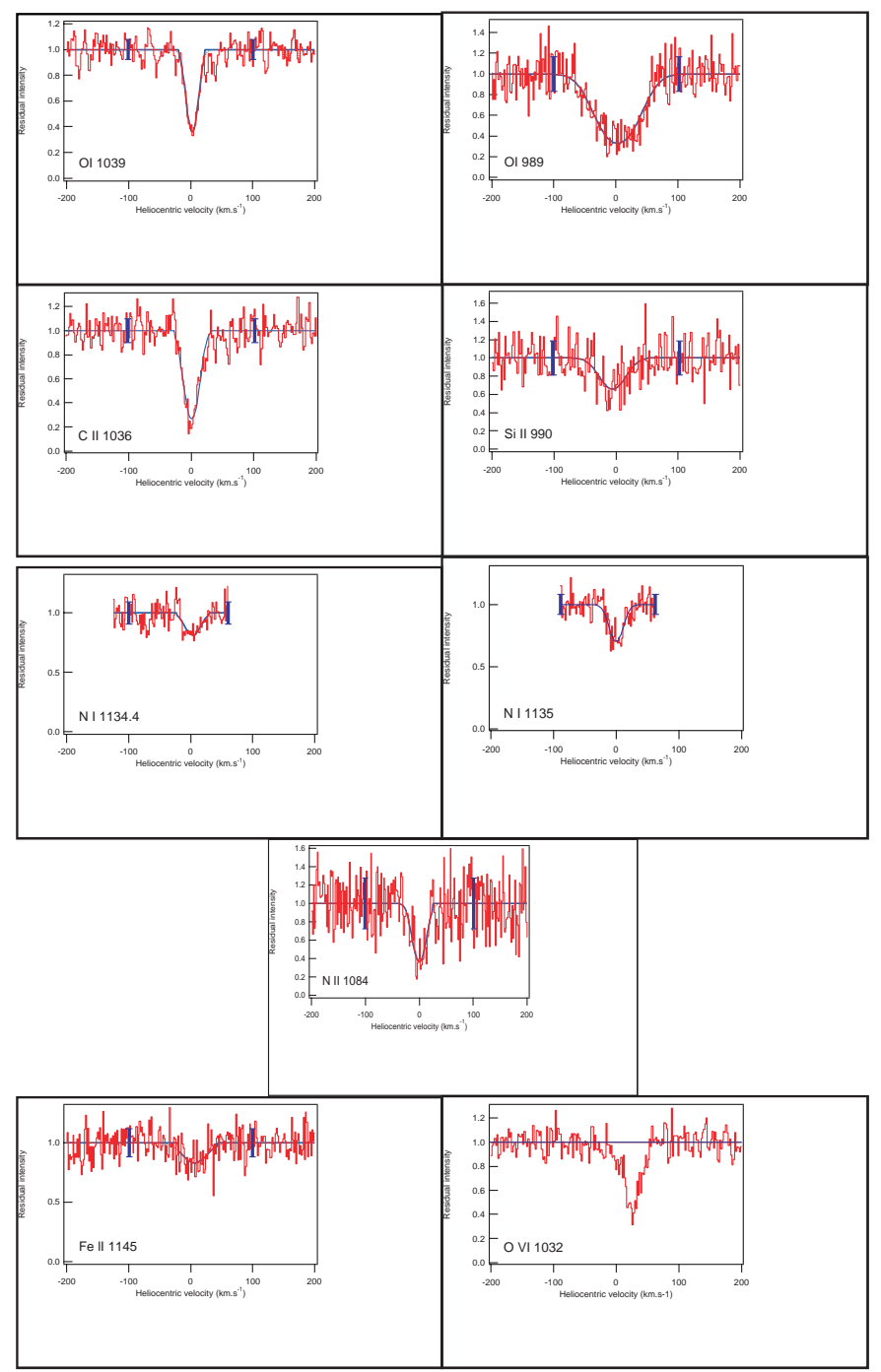

Fig. 2. Far ultraviolet interstellar absorption-line residual intensities and their best-fit profiles for REJ 1032+532. Solid bars indicate typical error sizes to the continuum level fits. Note that the O VI $\lambda 1032 \AA$ line is photospheric in origin.

(i) The equivalent width of the $\lambda 1032 \AA$ line is $\sim 69 \mathrm{~m} \AA$ (with a corresponding $\log N(\mathrm{O} \mathrm{VI})=13.7 \mathrm{~cm}^{-1}$ ), which is an order of magnitude greater than the marginal detection limits found for this ion in a study of 11 lines-of-sight in the local ISM by Oegerle et al. (2000). An interstellar O VI line of this strength is more compatible with that found at distances $>1 \mathrm{kpc}$ as determined from the FUSE survey of galactic O VI by Savage et al. (2001). (ii) The observed FUSE shift of both lines from their rest wavelength is consistent with that of all the other stellar lines in this spectrum (see Fig. 2). In particular, the value of this wavelength shift is identical to that of the strong C III stellar line sextuplet around $\lambda 1175 \AA$, whose photospheric origin has been confirmed by Holberg et al. (1999b). It was initially somewhat surprising that a (relatively cool) $47000 \mathrm{~K} \mathrm{DA}$ white dwarf could produce this anomalously high level of photospheric O VI absorption, but confirmation of this stellar line in the FUV spectra of several other (cool) white dwarfs has recently been made by Oliveira et al. (2001).

Finally we note that there is a (noise) feature in the blue wing of the aforementioned O VI $(\lambda 1032 \AA)$ line close to the expected rest wavelength of the O VI interstellar line (see Fig. 2). However, this absorption feature is not observed in the O VI $\lambda 1038 \AA$ line and thus cannot be confirmed as a real detection. However, its presence enables us to place a firm upper limit of $\log N(\mathrm{O} \mathrm{VI})<13.0 \mathrm{~cm}^{-2}$ for interstellar $\mathrm{O} \mathrm{VI}$ along this line-of-sight (see Table 1).

\section{Interstellar emission line analysis}

Although the resultant $S / N$ of the spectra are low (due to the relatively short exposure times), weak emission line features near $\lambda 1032 \AA$, which we associate with O VI ion emission, can be clearly seen in the night-time spectra of both " $A$ " and " $B$ " sight-lines. Using methods similar to those presented in Dixon et al. (2001), we have used IDL software routines to fit these O VI emission profiles by convolving the FUSE instrumental function (here estimated to be a $\sim 106 \mathrm{~km} \mathrm{~s}^{-1}$ "top-hat" function) with a best-fit Gaussian of $150 \mathrm{~km} \mathrm{~s}^{-1} F W H M$ for sightline " $A$ " and of $210 \mathrm{~km} \mathrm{~s}^{-1}$ for sight-line "B". The emission flux determined for the O VI $(\lambda 1032 \AA)$ line is $2500 \pm 700 \mathrm{LU}$ (a line unit, LU, is photons $\mathrm{cm}^{-2} \mathrm{~s}^{-1} \mathrm{sr}^{-1}$ ) for sight-line " $\mathrm{A}$ " and $3300 \pm 1100$ LU for sight-line "B". We quote one-sigma formal errors in these fluxes such that the two O VI lines have been detected with a significance level of 3.4- $\sigma$ for sight-line "A" and 3.1- $\sigma$ for sight-line "B". We have measured the central 


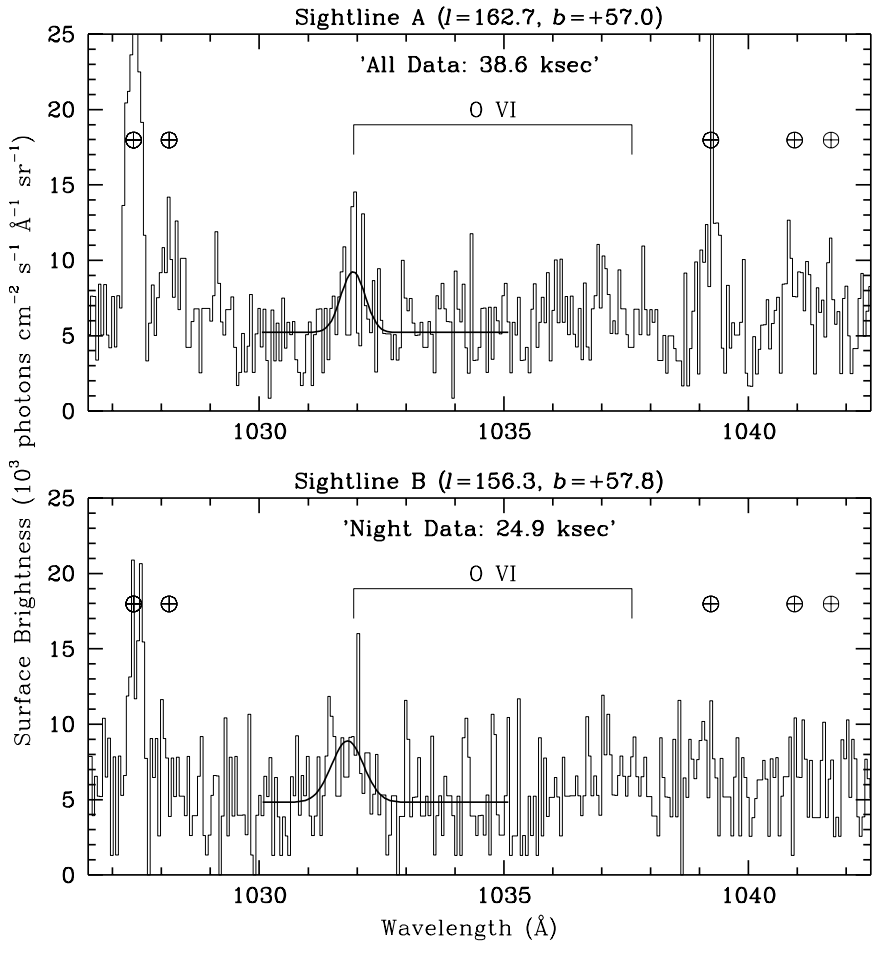

Fig. 3. Upper panel: far ultraviolet emission-line spectrum of the sight-line "A" $\left(l=162.7^{\circ}, b=+57.0^{\circ}\right)$. All recorded data are shown. Telluric emission lines are marked with the symbol " $\bigoplus$ "; and Lower panel: far UV emission line spectrum of the line-of-sight "B" $\left(l=156.3^{\circ}, b=+57.8^{\circ}\right)$. Night-only data are shown. The important O VI $\lambda 1032 \AA$ emission line can be clearly seen in both spectra. Models with parameters described in the text are shown by bold lines.

velocity for both of the OVI emission lines to be $V_{\text {helio }}=$ $-20 \pm 22 \mathrm{~km} \mathrm{~s}^{-1}$ and $V_{\text {helio }}=-55 \pm 30 \mathrm{~km} \mathrm{~s}^{-1}$ for sight-lines "A" and "B" respectively (add $4 \mathrm{~km} \mathrm{~s}^{-1}$ to obtain the equivalent LSR velocities). We note that the FUSE instrument is not ideally suited for obtaining diffuse emission-line measurements and the derived wavelength scale accuracy is not well constrained. Given the magnitude of our 1- $\sigma$ errors on the derived central velocity of the OVI lines, our results are consistent with both of the OVI emission lines being formed at approximately the same, slightly negative velocity when compared with the local standard of rest. For comparison we note that the bulk of neutral hydrogen in the Ursa Major region as sampled by $21 \mathrm{~cm}$ radio observations is dominated by two components of $F W H M 25 \mathrm{~km} \mathrm{~s}^{-1}$ near $V_{\text {helio }}=-5$ and $-55 \mathrm{~km} \mathrm{~s}^{-1}$ (Jahoda et al. 1990). Thus, our FUSE observations of the OVI emitting gas are consistent with an overall negative velocity flow in this general galactic direction.

The O VI $\lambda 1037.6 \AA$ line is intrinsically weaker than $\lambda 1032 \AA$ and is made even more difficult to detect due to profile contamination by the nearby CII $\lambda 1037 \AA$ line. However, we report a $3-\sigma$ upper limit value for the O VI $\lambda 1038 \AA$ line in sight-line "A" of $<2100 \mathrm{LU}$, and $<3000 \mathrm{LU}$ for sight-line "B". Similarly, we have been able to determine a marginal (2.5$\sigma)$ detection for the astrophysically interesting C III ( $\lambda 977 \AA)$ emission line of $5200 \pm 2000$ LU for sight-line "A" (based on the night-only data). Such a value is consistent with the only other (two) FUSE detections of this line by by Dixon et al. (2001). No meaningful upper limit can be determined for the C III line in the very low $\mathrm{S} / \mathrm{N}$ spectrum of sight-line "B".

\section{Discussion}

\subsection{Line-of-sight absorption}

The DA white dwarf, REJ $1032+532$, is located within an extension of the rarefied Local Bubble region that reaches out from the galactic disk towards the lower galactic halo (see Fig. 4). A physical boundary to this neutral gas-free interstellar chimney (if one exists) has yet to be accurately determined, but EUV data suggest that low values of neutral HI column density exist towards targets with a distance of at least $\sim 400 \mathrm{pc}$ in this direction (see Sect. 5.2). Although the LB is widely believed to contain hot and highly ionized interstellar gas, the detection of such gas in absorption has remained elusive (Oegerle et al. 2000). Although photoionization of the nearby $(d<5 \mathrm{pc})$ Local Interstellar Cloud (LIC) can seemingly account for the low levels of interstellar CIV and SiIV observed towards the star $\epsilon$ CMa (Gry \& Jenkins 2001; Slavin \& Frisch 2002), the failure to detect significant amounts of interstellar Si IV, C IV and O VI absorption towards all other lines-of-sight within the LB region suggests that the major local contribution from these high ions (which are all routinely detected towards more distant sight-lines) is most probably due to their formation at a conductive interface with the neutral boundary of the LB. We also note that alternative theories that invoke a far lower temperature for the LB gas due to extreme non-equilibrium conditions have also been forwarded to explain this apparent lack of high ion absorption detection (Breitschwerdt \& Schmutzler 1994).

Our FUSE spectrum of REJ $1032+532$ reveals only 8 detections of interstellar absorption lines (see Table 1). The ionization state of the interstellar plasma in which these far UV ions are formed is presently governed by the detection of the NII absorption line at $\lambda 1084 \AA$ (ionization potential $29.6 \mathrm{eV}$ ). This ionization level provides a natural explanation to the nondetection of the high ionization interstellar CIV and SiIV absorption lines towards REJ $1032+532$. Holberg et al. proposed that the bulk of the observed neutral interstellar gas absorption detected towards REJ $1032+532$ is formed in the local interstellar cloud (LIC) within $5 \mathrm{pc}$ of the Sun, such that the remaining $100 \mathrm{pc}$ line-of-sight to the white dwarf star is of extremely low neutral gas density. Unfortunately, due to the modest spectral resolution of the FUSE spectrograph and the limited number of IS lines presently detected, the derived best-fit doppler broadening parameters, $b$, listed in Table 1 do not provide very meaningful limits to the temperature of the absorbing interstellar gas in this direction. Finally, we note Holberg's anomalous detection of the (presumed) interstellar SiIII line ( $\lambda 1206 \AA$ ) with an ionization potential of $16.3 \mathrm{eV}$, the possible origin of which we discuss later in Sect. 5.2.

Holberg et al. (1999a) have determined a total hydrogen column density, $N(\mathrm{H})=N(\mathrm{HI})+N(\mathrm{HII})$, for this line-ofsight of $\log N(\mathrm{H})=18.87 \pm 0.06 \mathrm{~cm}^{-2}$. They also calculate that $N(\mathrm{HI}) \sim N(\mathrm{HII})$, indicating there are similar amounts of 
neutral and ionized hydrogen gas in this sight-line. Such a relatively low total hydrogen column density is typical for many other sight-lines within the LB cavity (Frisch 1998). Using this value of $N(\mathrm{H})$ we can derive gas-phase abundances for the elements of $\mathrm{C}, \mathrm{N}, \mathrm{O}, \mathrm{Si}$ and Fe relative to that of hydrogen using the total ion column densities listed in Table 1 (assuming that those ions listed are the dominant state of these elements in the local ISM). We note that although both the OI and NI ions are tightly coupled to HI gas by charge exchange reactions, they can (and do) exist in ionized HII regions (Sembach \& Savage 1996). For nitrogen we have detected lines from both NI and NII and thus the true elemental abundance of $N$ relative to $N(\mathrm{H})$ can be derived. However, for oxygen only the OI absorption lines are available and thus the derived abundance of $\mathrm{O}$ (which ignores the contribution from OII) is only an approximation. Ignoring the effects of ionization of this ion can typically result in a an error of \pm 0.15 dex in the presently derived abundance of $\mathrm{O}$.

For the sight-line towards REJ $1032+532$ we find $\log (\mathrm{C} / \mathrm{H})=-4.86, \log (\mathrm{N} / \mathrm{H})=-4.70, \log (\mathrm{O} / \mathrm{H})=-3.94$, $\log (\mathrm{Si} / \mathrm{H})=-5.25$ and $\log (\mathrm{Fe} / \mathrm{H})=-5.44$ for these elements. Apart from our derived value of $\log (\mathrm{C} / \mathrm{H})$, such element abundance values are within 0.2 dex of those found by Holberg et al. (1999a) towards this star, who also made abundance comparisons with a total value of $N(\mathrm{H})$. Our findings also support the corresponding pattern of elemental depletion that characterizes interstellar sight-lines through the LIC to other stars in the Local Bubble (Vidal-Madjar et al. 1998; Jenkins et al. 2000). We note that our value of $\log (\mathrm{C} / \mathrm{H})$ is 1.0 dex lower than that found by Holberg et al. and this difference may be due to line saturation effects of the CII $\lambda 1334 \AA$ line used by Holberg et al. in their curve-of-growth column density derivation. Recently Moos et al. (2002) have investigated the interstellar abundances of $\mathrm{OI} / \mathrm{HI}$ and NI/HI for lines-of-sight towards 7 white dwarf stars within $180 \mathrm{pc}$ of the Sun and found average relative abundances of $\log (\mathrm{OI} / \mathrm{HI})=-3.47$ and $\log (\mathrm{NI} / \mathrm{HI})=-4.41$, both values being $\sim 0.5$ dex higher than our abundances derived for the REJ 1032+532 sight-line (which include the contribution of HII gas). Moos et al. find that the variability of the OI/NI ratio in the local ISM is largely due to the variation in the local intensity of ionizing radiation that in turn affects the relative ionization balance of NI (i.e. a significant fraction of $\mathrm{N}$ resides in the form of NII, as confirmed by our present detection of the NII line).

In summary, our FUSE observations of the absorption profiles of gas in the interstellar direction towards REJ $1032+532$ support the findings of Holberg et al. (1999a) in which the bulk of absorption resides in a single interstellar cloud, presumed to be the LIC, at a velocity of $V_{\text {helio }}=+0.4 \mathrm{~km} \mathrm{~s}^{-1}$. In addition, the FUV absorption profiles show no evidence that REJ $1032+532$ lies beyond a cold, dense neutral boundary to the Local Bubble, in accord with the boundary contours of the LC feature as defined by Sfeir et al. (1999), and shown in Fig. 4. Additionally, we find no signs of the confined, cool $(T<500 \mathrm{~K})$ layer of neutral gas that generally defines the inner galactic halo in most other galactic directions, nor have we detected signs of the warmer $(T \sim 6000 \mathrm{~K})$ more diffuse gas of the Lockman Layer (Lockman 1984). Thus, if all of the neutral interstellar

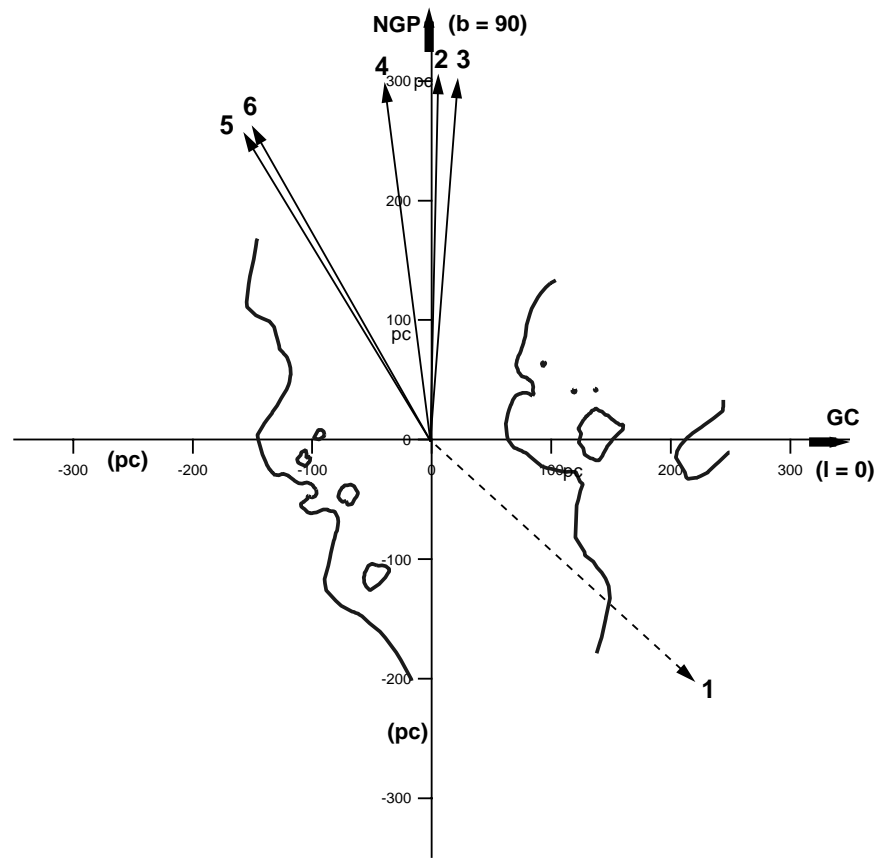

Fig. 4. Plot of the neutral gas boundary of the Local Bubble in the Meridian Plane with the 6 lines-of-sight in which O VI $\lambda 1032 \AA$ has been detected by the FUSE satellite are shown. See Table 2 for information on the labelled sight-lines. Note that due to the map projection selected, the direction of sight-line 1 shown is only approximate since it points out of the paper.

absorption observed towards REJ $1032+532$ is associated with the LIC (i.e. within $10 \mathrm{pc}$ ) then the remaining $100 \mathrm{pc}$ of the interstellar medium between the LIC and the white dwarf is an ionized region with $\log N(\mathrm{HII})=18.52 \mathrm{~cm}^{-2}$ (Holberg et al. 1999a).

\subsection{Line-of-sight emission}

Both the "A" and "B" sight-lines sample emitting gas located in an extension of the LB cavity that extends towards the lower galactic halo. To date there have been four other measurements of diffuse O VI emission recorded by the FUSE satellite, all taken towards lines-of-sight with similarly high galactic latitudes $>|40|^{\circ}$ (Shelton et al. 2001; Dixon et al. 2001; Shelton 2002). In Table 2 we summarize all relevent information concerning these six O VI emission observations by FUSE. We also list estimates of the ROSAT line-of-sight SXRB for the summed $R 1$ and $R 2$ bands averaged over a radius of $0.4^{\circ}$ (see the NASA HEASARC X-Ray Background Tool web-page at http://heasarc.gsfc.nasa.gov/cgi-bin/Tools/xraybg/xraybg.pl, which uses the data of Snowden et al. 1997).

The directions of these, and the present " $A$ " and "B" sightlines, in relation to the neutral boundary of the Local Bubble are shown in Fig. 4. This neutral boundary contour has been defined by an interstellar line-of-sight $\mathrm{NaI}$ D2-line equivalent width $>50 \mathrm{m \AA}$ (Sfeir et al. 1999). A NaI D2-line of this strength corresponds to a neutral hydrogen column density $>10^{20} \mathrm{~cm}^{-2}$, which provides an optical depth of at least unity for a $0.25 \mathrm{keV}$ soft X-ray photon. Note that both "ends" of the high latitude extensions to the LB are, as yet, undefined by the $\mathrm{NaI}$ 
observations (due to a dearth of suitable stellar targets). Thus, at present we are unable to estimate with great confidence if (and at what distance) these sight-lines encounter a plausible neutral boundary to the LB. However, a neutral boundary to the LB should exist in the northern hemisphere direction (at some, as yet undetermined distance) since values of the hydrogen column density, $N(\mathrm{HI})$, derived from $21 \mathrm{~cm}$ observations along sight-lines "A" and "B" are both of a similar (high) value of $N(\mathrm{HI}) \sim 2 \times 10^{20} \mathrm{~cm}^{-2}$ (Dickey \& Lockman 1990). Additionally, we note that in the galactic maps of the distribution of sources detected by the EUVE satellite, there is only one direction at positive galactic latitudes in which sources (hot white dwarfs) were detected to distances $>150 \mathrm{pc}$ (Welsh et al. 1999). This direction covers a relatively small area of $7^{\circ}$ radius centered on $\left(l=162^{\circ}, b=+58.0^{\circ}\right)$ and contains EUV detections of 3 white dwarfs with distances (taken from Vennes et al. 1997) of $230 \mathrm{pc}$ (REJ 1043+490), $316 \mathrm{pc}$ (REJ 1059+514) and 404 pc (REJ 1043+449). Since the ability to detect EUV sources is critically dependent on the line-of-sight neutral hydrogen column density, we can confidently place a minimum distance to the neutral boundary to the LB in this one particular direction at $\sim 400 \mathrm{pc}$. Both of our " $\mathrm{A}$ " and "B" sight-lines are within $\sim 5^{\circ}$ of this particular direction and they are similarly close to the $100 \mathrm{deg}^{2}$ field in Ursa Major that has the least neutral hydrogen column density of any direction in the sky (Jahoda et al. 1990).

It is immediately apparent from the information listed in Table 2 that the intensity of diffuse emission from the O VI $\lambda 1032 \AA$ line recorded by the FUSE instrument is remarkably similar for all six directions thus far sampled. This implies a fairly constant distribution of OVI surface brightness at high galactic latitudes of about $2700 \mathrm{LU}$ with a standard deviation of 450 LU. All of these sight-lines sample emission from high galactic latitudes and thus it is still unknown if the same levels of O VI surface brightness apply in sight-lines that sample emission from only the galactic disk. Clearly further FUSE observations are required to resolve this point, and to determine if a possible sight-line selection bias currently exists for these six observations. Absorption measurements of O VI towards high latitude sight-lines show a relatively large variation (of about an order of magnitude) in the measured O VI column density values (Savage et al. 2000), such that the majority of O VI absorption probably originates in a (highly) variable contribution from the hot halo gas. However, in contrast, the relative low levels of variability in the presently determined FUSE O VI emission intensities would argue for a more local origin in which there is little contribution from halo O VI ions. Therefore, bearing in mind the small number of sight-lines thus far sampled, the present observations of constancy of O VI emission intensity would seem to favor its production at a local interface, which we presently identify as the (thin) neutral boundary zone to the Local Bubble.

Shelton et al. (2001) have used the observed intensity of $\mathrm{O}$ VI emission to a halo sight-line (which is of a very similar intensity value to our present findings) to estimate the column density, intrinsic intensity, electron density, thermal pressure and depth of the emitting hot gas. Their predicted O VI intensities are too large for the observed emission to originate solely in the hot Local Bubble, and they favor some additional O VI emission forming in the galactic thick disk and/or lower halo region. Also, if all of the emission came from just the LB then the thermal pressure of the O VI emitting gas would be $P / k \sim 80000 \mathrm{~K} \mathrm{~cm}^{-3}$, contrary to the far lower observed pressure values of $\sim 2200 \mathrm{~K} \mathrm{~cm}^{-3}$ for the LIC (Lallement 1998) and $\sim 10000 \mathrm{~K} \mathrm{~cm}^{-3}$ for the hot LB gas found from the emission measure of the SXRB emission (Snowden et al. 1990). Also the calculated emitting depth of the O VI emission seems to be small $(<1 \mathrm{pc})$, suggesting that the O VI-bearing gas fills a small volume which is consistent with it being formed at a conductive interface. We deem it highly unlikeley that OVI could be formed by photoionization processes in the LB since it would require a stellar ionizing flux of at least $114 \mathrm{eV}$ to form this ion.

The total ROSAT $0.25 \mathrm{keV} \mathrm{SXRB} \mathrm{R1} \mathrm{and} \mathrm{R2} \mathrm{(R12)} \mathrm{in-}$ tensities from the six sight-lines listed in Table 2 are very similar for 4 of the sight-lines (with an average of $\sim 1000 \times$ $10^{-6}$ counts $\mathrm{s}^{-1} \operatorname{arcmin}^{-2}$ ), but are a factor $\sim 5$ higher towards the two sight-lines sampled by Dixon et al. (2001). We note that these latter two sight-lines are very close to the Coma and Virgo Clusters, which accounts for both of their anomalously high observed SXRB count-rates. It has generally been assumed that the emission from the million $\mathrm{K}$ degree soft $\mathrm{X}$-ray emitting plasma, whose distribution has been demonstrated to be anti-correlated with that of neutral HI gas, should correlate with the $\mathrm{O}$ VI emitting gas. Although the results shown in Table 2 clearly show a constancy in the emission levels of both O VI and the R12 SXRB flux (for the 4 sight-lines), we are unable at present to show that there is an actual correlation between these two fluxes. Future FUSE O VI observations towards galactic regions with associated anomalously high and very low SXRB fluxes would perhaps resolve this point.

In a recent re-analysis of the ROSAT SXRB survey data, Kuntz \& Snowden (2000) have shown that at least 3 sources are required to explain the observed distribution of the $0.1-1 \mathrm{keV}$ flux: (i) a hot LB contribution, (ii) an extragalactic contribution and (iii) a galactic contribution that resides outside of the galactic disk ISM. Using their model we list the estimated SXRB R12 count rates that can be attributed solely to emission from the presumed million degree K Local Hot Bubble gas in the last column of Table 2 (K. D. Kuntz, private communication). We note that this local contribution to the SXRB flux is variable and is not correlated with the measured intensities of the OVI emission lines. Parenthetically, we also note that the OVI emission line intensity is aslo uncorrelated with the remainder of the SXRB flux that the Kuntz and Snowden model attribute to emission sources residing beyond the galactic disk.

Finally, we suggest that the local conductive interface at the LB boundary that we favor to be responsible for the similar levels of observed O VI emission surface brightness may also be responsible for producing the observed levels of local O VI absorption. If the majority of local O VI absorption is formed at a neutral LB interface, then this could well explain the sudden increase in the levels of O VI absorption measured towards targets with distances in excess of the LB boundary. It would also explain our present non-detection of O VI absorbing gas towards REJ $1032+532$ and also the similar non-detections by 
Table 2. FUSE O VI emission line observational data and ROSAT soft X-ray background fluxes.

\begin{tabular}{ccccc}
\hline \hline Direction $(l, b)$ & $\begin{array}{c}\text { O VI } \lambda 1032 \AA \text { Intensity } \\
\left.\text { (photons cm } \mathrm{s}^{-1} \mathrm{sr}^{-1}\right)\end{array}$ & O VI Reference & $\begin{array}{c}\text { ROSAT R12 SXRB Flux } \\
\left(10^{-6} \mathrm{counts} \mathrm{s}^{-1} \operatorname{arcmin}^{-2}\right)\end{array}$ & $\begin{array}{c}\text { LHB SXRB Flux* } \\
\left(10^{-6} \mathrm{counts} \mathrm{s}^{-1} \mathrm{arcmin}^{-2}\right)\end{array}$ \\
\hline $1:\left(315.0^{\circ},-41.3^{\circ}\right)$ & $2930 \pm 290$ & Shelton et al. (2001) & 930 & 450 \\
$2:\left(57.6^{\circ},+88.0^{\circ}\right)$ & $2000 \pm 600$ & Dixon et al. (2001) & 4435 & 760 \\
$3:\left(284.2^{\circ},+74.5^{\circ}\right)$ & $2900 \pm 700$ & Dixon et al. (2001) & 5530 & 385 \\
$4:\left(113.0^{\circ},+70.7^{\circ}\right)$ & $2580 \pm 380$ & Shelton (2002) & 1000 & 500 \\
$5:\left(162.7^{\circ},+57.0^{\circ}\right)$ & $2500 \pm 700$ & This Paper (“A”) & 1130 & 730 \\
$6:\left(156.3^{\circ},+57.8^{\circ}\right)$ & $3300 \pm 1100$ & This Paper ("B”) & 1140 & 870 \\
\hline$*$ Derived from the Kuntz E Snowden (2000) model of the Local Hot Bubble. \\
\hline
\end{tabular}

FUSE towards other targets with distances within the LB neutral boundary (Oegerle et al. 2000).

Alternatively, a more radical explanation for our present results could be that there is no hot million degree $\mathrm{K}$ gas within the LB cavity and the SXRB emission arises entirely at the LB interface and beyond. In this extreme scenario the LB gas would possess a temperature of only $\sim 40000 \mathrm{~K}$, as suggested by the non-collisional ionization model of Breitschwerdt \& Schmuztler (1994). In such a model a low pressure of $P / k \sim$ $2000 \mathrm{~cm}^{-3} \mathrm{~K}$ for the LB can be explained, as can the observed upper limits for the high ionization absorption lines of C IV, N V and O VI for nearby sight-lines (but only under the assumption of an element depleted local interstellar gas). However, more recent calculations by Brietschwerdt (2001) predict very strong emission from the C III $\lambda 977 \AA$ line, which is inconsistent (by an order of magnitude) with the marginal detections of this line found presently by us, as well as by other authors towards the sight-lines listed in Table 2. We note that such a "warm" gas LB model could account for the anomalously high levels of interstellar SiIII ( $\lambda 1206 \AA)$ observed towards $\beta$ CMa (Dupin \& Gry 1998), $\epsilon$ CMA (Gry \& Jenkins 2001), REJ 1032+532 (Holberg et al. 1999a), and several other nearby hot white dwarfs (Holberg et al. 1998). However the interstellar origin of these anomalously strong SiIII lines is still in some doubt, which has led some authors to speculate that they could be of stellar or circumstellar origin.

In conclusion, we note that any theory of a hot or warm gas LB cavity must now also explain the presently identified constancy of both O VI and total SXRB emission intensities for the lines-of-sight $>|40|^{\circ}$ thus far sampled by FUSE, in addition to explaining the low thermal pressure values and the seeming absence of high ionization absorption lines in the local interstellar medium.

\section{Conclusion}

We have carried out far ultraviolet absorption observations towards the nearby hot white dwarf star REJ $1032+532(d=$ $116 \mathrm{pc}$ ), together with observations of the far UV emission from two nearby lines-of-sight using the NASA FUSE satellite. All three sight-lines are within $\sim 5^{\circ}$ on the sky and they form part of an interstellar feature called the Local Chimney which is an extension of the rarefied Local Bubble cavity at high positive latitudes that could reach further than $\sim 400 \mathrm{pc}$ away from the galactic plane.
We have detected only eight interstellar absorption lines in the far UV spectrum $(\lambda 912-1180 \AA)$ of the hot white dwarf star REJ $1032+532$. No high ionization O VI $\lambda 1032 \AA$ absorption has been detected (to an upper limit of $N(\mathrm{O} \mathrm{VI})<13.00 \mathrm{~cm}^{-2}$ ), which is consistent with the non-detections of other high ions such as CIV and SiIV reported by Holberg et al. (1999a) towards this star. The detection of interstellar NII towards this white dwarf presently sets an upper limit of $29.6 \mathrm{eV}$ to the ionization potential of gas in this sight-line. Using absorption line-profile fitting techniques we have derived best-fit ion column densities for all eight lines and compared these with the total hydrogen column, $\log N(\mathrm{H})=18.87 \mathrm{~cm}^{-2}$, measured towards this star. Abundances of $\mathrm{C}, \mathrm{N}, \mathrm{O}, \mathrm{Si}$ and $\mathrm{Fe}$ with respect to hydrogen have been derived, and all are consistent with the elemental abundances found by several authors for other sight-lines in the local ISM that pass through the Local Interstellar Cloud $(d<5 \mathrm{pc})$. No evidence is found to support REJ $1032+532$ lying beyond the neutral boundary to Local Bubble, or that it resides in a warm diffuse neutral medium similar to the lower halo Lockman Layer.

Far ultraviolet diffuse background emission has been detected in the high ionization line of O VI $\lambda 1032 \AA$ for both lines of sight observed. In the direction of sight-line "A" $\left(l=162.7^{\circ}\right.$, $\left.b=+57.0^{\circ}\right)$ we have detected an O VI $\lambda 1032 \AA$ emission line intensity of $2500 \pm 700 \mathrm{LU}$ and for sight-line "B" $\left(l=156.3^{\circ}\right.$, $b=+57.8^{\circ}$ ) we have recorded an intensity of $3300 \pm 1100 \mathrm{LU}$. These two O VI line intensity levels are remarkably similar to the levels of emission found by other authors for the other (four) directions sampled thus far by the FUSE satellite. The present average intensity of O VI $\lambda 1032 \AA$ emission to all six sight-lines is $2700 \mathrm{LU}$ with a standard deviation of $450 \mathrm{LU}$. The apparent similarity of O VI emission level intensity may be understood if this ion is formed locally, at a conductive interface with the neutral boundary to the Local Bubble region. This may also be the site for the production of the observed absorption due to the O VI ion, which has thus far been detected by the FUSE satellite only towards targets with distances beyond the LB neutral boundary.

The observed $0.25 \mathrm{keV}$ total SXRB R12 flux intensities from four of these sight-lines are also similar $(\sim 1000 \times$ $10^{-6}$ counts s $\left.^{-1} \operatorname{arcmin}^{-2}\right)$. However, the soft X-ray contribution from emission originating locally in the million degree $\mathrm{K}$ gas of the Local Hot Bubble (as modelled by Kuntz \& Snowden $2000)$ is highly variable and is uncorrelated with the corresponding FUSE OVI line intensities. 
A model of the hot gas in which all of the observed O VI intensity originates in the Local Bubble predicts a thermal pressure for the hot gas that is several times larger than that estimated from the SXRB observations. Even though a low LB pressure and the observed anomalous levels of SiIII absorption can be explained by a "warm" $(T \sim 40000 \mathrm{~K})$, noncollisionally ionized gas, this model is rejected due to the observed low levels of C III $\lambda 977 \AA$ emission, which is contrary to the far higher levels predicted by the model of Breitschwerdt (2001). In summary, any new theory of hot gas in the Local Bubble will have to explain not only the observed low levels of thermal pressure, but also the presently identified constancy in the levels of both O VI and total SXRB emission observed at galactic latitudes $>|40|^{\circ}$.

Acknowledgements. This work is based on data obtained under the NASA FUSE Guest Investigator Program. The NASA-CNES-CSA FUSE Mission is operated by the Johns Hopkins University. Financial support to U.S. particpants has been provided by the NASA contract NAS5-32985. We thank Dr. John Vallerga for very helpful discussions, and we also thank Dr. K. D. Kuntz for his soft X-ray model predictions for the Local Hot Bubble gas emission.

\section{References}

Breitschwerdt, D., \& Schmutzler, T. 1994, Nature, 371, 774

Breitschwerdt, D. 2001, Ap\&SS, 276, 163

Cox, D., \& Smith, B. 1974, ApJ, 189, L105

Dickey, J., \& Lockman, F. 1990, ARA\&A, 28, 215

Dixon, W. V., Hurwitz, M., Jelinsky, P., et al. 1998, BAAS, 193, 1208

Dixon, W. V., Sallmen, S., Hurwitz, M., \& Lieu, R. 2001, ApJ, 552, L69

Dupin, O., \& Gry, C. 1998, A\&A, 335, 661

Edelstein, J., Korpela, E., Dixon, W. V., et al. 2000, BAAS, 197, 1413

Frisch, P. 1998, in The Local Bubble and Beyond, IAU Coll. 166, Lect. Notes Phys., 506, 269

Gry, C., \& Jenkins, E. 2001, A\&A, 367, 617

Holberg, J., Barstow, M., \& Sion, E. 1998, ApJS, 119, 207

Holberg, J., Bruhweiler, F., Barstow, M., \& Dobbie, P. 1999, ApJ, 517, 841
Holberg, J., Barstow, M., Bruhweiler, F., et al. 1999, ApJ, 517, 850

Jahoda, K., Lockman, F., \& McCammon, D. 1990, ApJ, 354, 184

Jenkins, E., Oegerle, W., Gry, C., et al. 2000, ApJ, 538, L81

Kuntz, K., \& Snowden, S. 2000, ApJ, 543, 195

Lallement, R. 1998, in The Local Bubble and Beyond, IAU Coll. 166, Lect. Notes Phys., 506, 19

Lockman, F. 1984, ApJ, 283, 90

Lockman, F., Jahoda, K., \& McCammon, D. 1986, ApJ, 302, 432

McKee, C., \& Ostriker, J. 1977, ApJ, 218, 148

Moos, H. W., Cash, W., Cowie, L., et al. 2000, ApJ, 538, L1

Moos, H. W., Sembach, K., Friedman, S., et al. 2002, ApJS, 140, 3

Oegerle, W., Jenkins, E., Shelton, R., et al. 2000, BAAS, 197, 07.18

Oliveira, C., Chayer, P., Dupuis, J., \& Moos, H. W. 2001, BAAS, 199, 1710

Sahnow, D., Moos, H. W., Ake, T., et al. 2000, ApJ, 538, L7

Sfeir, D. M. 1999, Ph.D. Thesis, University of Paris 6

Savage, B., Semach, K., \& Lu, L. 1997, AJ, 113, 2158

Savage, B., Sembach, K., Jenkins, E., et al. 2000, ApJ, 538, L27

Savage, B., Sembach, K., Wakker, B., et al. 2001, BAAS, 199, 6504

Schmutzler, T., \& Tscharnuter, W. 1993, A\&A, 273, 318

Sembach, K., \& Savage, B. 1996, ApJ, 457, 211

Sfeir, D. M., Lallement, R., Crifo, F., \& Welsh, B. Y. 1999, A\&A, 346, 785

Shapiro, P., \& Field, G. 1976, ApJ, 205, 762

Shelton, R., Kruk, J., Murphy, E., et al. 2001, ApJ, 560, 730

Shelton, R. 2002, ApJ, 569, 758

Slavin, J., \& Frisch, P. 2002, ApJ, 565, 364

Snowden, S., Cox, D., McCammon, D., \& Sanders, W. 1990, ApJ, 354,211

Snowden, S., Hasinger, G., Jahoda, K., et al. 1994, ApJ, 430, 601

Snowden, S., Egger, R., Freyberg, M., et al. 1997, ApJ, 485, 125

Snowden, S., Egger, R., Finkbeiner, D., et al. 1998, ApJ, 493, 715

Spitzer, L. 1956, ApJ, 124, 20

Vallerga, J. V. 1996, Space Sci. Rev., 78, 277

Vennes, S., Thejll, P., Galvan, R. G., \& Dupuis, J. 1997, ApJ, 480, 714

Vidal-Madjar, A., Lemoine, M., Ferlet, R., et al. 1998, A\&A, 338, 694

Welsh, B. Y., Craig, N., Vedder, P., \& Vallerga, J. 1994, ApJ, 437, 638

Welsh, B. Y., Sfeir, D., Sirk, M., \& Lallement, R. 1999, A\&A, 352, 308

Welsh, B. Y., Sfeir, D., Sallmen, S., \& Lallement, R. 2001, A\&A, 372, 516 